\title{
Screening of the SLC17A8 gene as a causative factor for autosomal dominant non-syndromic hearing loss in Koreans
}

Nari Ryu ${ }^{1 \dagger}$, Borum Sagong ${ }^{1 \dagger}$, Hong-Joon Park ${ }^{2 \dagger}$, Min-A Kim ${ }^{1,3}$, Kyu-Yup Lee $^{4}$, Jae Young Choi ${ }^{5}$ and Un-Kyung Kim ${ }^{1,3^{*}}$

\begin{abstract}
Background: One of the causes of sensorineural hearing loss (SNHL) is degeneration of the inner hair cells in the organ of Corti in the cochlea. The SLC17A8 (solute carrier family 17, member 8) gene encodes vesicular glutamate transporter 3 (VGLUT3), and among its isoforms (VGLUT1-3), only VGLUT3 is expressed selectively in the inner hair cells (IHCS). VGLUT3 transports the neurotransmitter glutamate into the synaptic vesicles of the IHCs. Mutation of the SLC17A8 gene is reported to be associated with DFNA25 (deafness, autosomal dominant 25), an autosomal dominant non-syndromic hearing loss (ADNSHL) in humans.

Methods: In this study, we performed a genetic analysis of 87 unrelated Korean patients with ADNSHL to determine whether the SLC17A8 gene affects hearing ability in the Korean population.

Results: We found a novel heterozygous frameshift mutation, 2 non-synonymous variations, and a synonymous variation. The novel frameshift mutation, p.M206Nfs*4, in which methionine is changed to asparagine at amino acid position 206, resulted in a termination codon at amino acid position 209. This alteration is predicted to encode a truncated protein lacking transmembrane domains 5 to 12. This mutation is located in a highly conserved region in VGLUT3 across multiple amino acid alignments in different vertebrate species, but it was not detected in 100 unrelated controls who had normal hearing ability. The results from our study suggest that the p.M206Nfs*4 mutation in the SLC17A8 gene is likely a pathogenic mutation that causes ADNSHL.
\end{abstract}

Conclusion: Our findings can facilitate the prediction of the primary cause of ADNSHL in Korean patients.

Keywords: DFNA25, SLC17A8, VGLUT3, Autosomal dominant non-syndromic hearing loss, Mutation

\section{Background}

Hearing loss is a relatively common defect, with an incidence of 1 to 3 per 1000 births $[1,2]$. It can be classified as sensorineural hearing loss (SNHL), conductive hearing loss, or mixed hearing loss, and the major cause of hearing loss is attributed to genetic or environmental factors [3, 4]. SNHL is usually caused by malfunction or degeneration of the cochlea, including the hair cells within the organ of Corti $[5,6]$.

\footnotetext{
* Correspondence: kimuk@knu.ac.kr

${ }^{\dagger}$ Equal contributors

'Department of Biology, College of Natural Sciences, Kyungpook National University, Daegu 41566, South Korea

${ }^{3}$ School of Life Sciences, BK21 Plus KNU Creative BioResearch Group, Kyungpook National University, Daegu 41566, South Korea

Full list of author information is available at the end of the article
}

Approximately $70 \%$ of hereditary SNHL cases are recognized as non-syndromic, which refers to hearing loss not accompanied by any clinical signs or symptoms [7-9]. Among non-syndromic hearing loss cases, approximately $75-80 \%$ of cases are defined as autosomal recessive (DFNB), $10-20 \%$ as autosomal dominant (DFNA), $1 \%$ as X-linked, and less than $1 \%$ as mitochondrial inheritance $[4,10]$. Autosomal dominant non-syndromic hearing loss (ADNSHL) is genetically heterogeneous, and 33 related genes have been reported to date (http://hereditaryhearingloss.org/). Patients with ADNSHL typically have the characteristics of a late-onset age, high-frequency sensorineural hearing loss, and progressive worsening with age $[11,12]$. Moreover, the causative gene of 
ADNSHL is considered to be a candidate gene for presbycusis $[11,13]$.

The SLC17A8 (solute carrier family 17 , member 8 ) gene associated with ADNSHL lies within the locus of DFNA25 (MIM: 605583), which is defined to be in the 20-cM region of chromosome 12q21-24 [11, 13]. The SLC17A8 gene encodes the vesicular glutamate transporter 3 (VGLUT3) protein, which consists of 589 amino acids in 12 exons [13]. This protein is predicted to be composed of 12 transmembrane helices, which are packed in 4 groups of 3 each [14]. Each transmembrane domain is composed of 21 amino acids; the region between amino acid positions 80 and 502 is defined as the major facilitator superfamily (MFS) [15] (http://www.ncbi.nlm.nih.gov/protein/ NP_647480.1). They are single-peptide secondary transporter proteins, which transport diverse hydrophilic solutes $[15,16]$. There are 3 VGLUT subtypes (VGLUT1, 2, and 3), of which only VGLUT3 is expressed in the inner hair cells (IHCs) [14, 17]. The IHCs are the primary auditory sensory receptors, and glutamate is the essential excitatory neurotransmitter in the IHCs [17-20]. The main function of VGLUT3 in the IHCs is to transport this neurotransmitter into vesicles [17]. In the absence of VGLUT3 expression, the uptake and release of glutamate are disrupted at the afferent synapses of the IHCs [17-19]. Therefore, mutations in the SLC17A8 gene would result in the malfunctioning of VGLUT3, which leads to ADNSHL $[13,17]$.

To date, only 1 mutation in the SLC17A8 gene, namely, p.A211V, has been reported, which was in candidate family members of Czech and German descent in the United States [13]. These subjects had progressive, high-frequency SNHL with an autosomal dominant inheritance pattern [13]. Because no such studies on the SLC17A8 gene in the Asian population have been reported to date, we performed gene screening in 87 unrelated Korean ADNSHL candidates and 100 unrelated Koreans with normal hearing to determine whether this gene should be considered as a contributing factor to ADNSHL in the Korean population.

\section{Methods}

\section{Subjects and clinical evaluation}

For this study, 87 unrelated subjects showing autosomal dominant inheritance in their pedigrees were recruited from the Department of Otorhinolaryngology-Head and Neck Surgery, Kyungpook National University Hospital in Daegu, Yonsei University Health System Hospital in Seoul, and Soree Ear Clinic in Seoul, Korea. This cohort was used in the previous genetic studies [21, 22]; these previous ones and this study were performed in the similar period. The hearing levels of the patients were examined by pure-tone audiometry (PTA). The average threshold in the PTA was measured at 500, 1000, 2000, and $4000 \mathrm{~Hz}$ in a sound-treated room. For PTA, the levels of hearing loss are described as follows: $\sim 20 \mathrm{~dB}$ is assigned as normal hearing; $21-40 \mathrm{~dB}$ as mild hearing impairment; $41-70 \mathrm{~dB}$ as moderate hearing impairment; 71-95 dB as severe hearing impairment; and $>95 \mathrm{~dB}$ as profound hearing impairment [23]. One hundred unrelated individuals with normal hearing were recruited from Kyungpook National University as normal controls. All the participants provided written informed consent before the study according to the protocol approved by the Ethics Committee of Kyungpook National University Hospital.

\section{Genetic analysis}

For genetic analysis, the genomic DNA of the 87 patients and 100 controls with normal hearing was isolated from blood samples using FlexiGene DNA kits (Qiagen, Hilden, Germany). All 12 exons and intron-exon boundaries of the SLC17A8 gene (GenBank ID: NM_139319.2) were amplified by polymerase chain reaction (PCR) using h-Taq DNA Polymerase (Solgent, Daejeon, Korea). Primers for each exon for PCR and direct sequencing were designed to be specific to their regions using Primer3web version 4.0.0 (http://primer3.ut.ee/). The amplified PCR products were then purified with exonuclease I (USB, Cleveland, OH, USA) and shrimp alkaline phosphatase (USB, Cleveland, OH, USA). Purified PCR products were then sequenced with the BigDye Terminator version 3.1 Cycle Sequencing Kit (Applied Biosystems, Foster City, CA, USA). The products were purified by ethanol precipitation and assayed with 3130xl Genetic Analyzer (Applied Biosystems, Foster City, CA, USA). The observed data were analyzed using Sequencing Analysis version 5.2 (Applied Biosystems, Foster City, CA, USA) and Chromas Lite version 2.1.1 (Technelysium Pty Ltd., Tewantin, QLD, Australia) software. Multiple sequence alignment was performed to compare the amino acid sequences of different vertebrate species, including Homo sapiens, Aotus nancymaae, Bos taurus, Mus musculus, Rattus norvegicus, Xenopus tropicalis, and Danio rerio, using CLC Sequence Viewer version 6.9 (CLC Bio, Aarhus, Denmark). The novelty of the newly detected variants was investigated using the dbSNP database (http://www.ncbi.nlm.nih.gov/snp/) and the 1000 Genomes Project Database (http://www.1000genomes.org/) as references. Further prediction of the pathogenicity was performed using the Mutation Taster program (http://www.mutationtaster.org).

\section{Restriction fragment length polymorphism analysis}

To confirm whether the variations were present in the 100 normal controls, we performed a restriction fragment length polymorphism (RFLP) analysis. The restriction enzymes FokI (Takara Bio Inc., Otsu, Shiga, Japan) 
and PshAI (New England Biolabs Inc., Beverly, MA, USA) were chosen using SNP-RFLPing (http://bio.kuas.edu.tw/snp-rflping2/rflpUI.jsp) and Webcutter version 2.0 (http://rna.lundberg.gu.se/cutter2/). Each product was treated with each of the restriction enzymes, and they were incubated at $37^{\circ} \mathrm{C}$ for $1 \mathrm{~h}$ to activate the enzyme. After $1 \mathrm{~h}$, the products were examined by electrophoresis on $2 \%$ agarose gels.

\section{Results}

In this study, we screened the SLC17A8 gene in 87 patients with ADNSHL and identified a novel frameshift mutation in 1 patient, 2 non-synonymous variations in 2 patients, and a synonymous variation in 18 patients. These patients with $S L C 17 A 8$ variations were negative for other mutations in the previous studies [21, 22].

The SD-38 family consisted of 3 generations, with 8 affected individuals, and the pattern of inheritance was autosomal dominant (Fig. 1a). The proband (III-2) of this family was a 47-year-old man with bilateral severe hearing loss, and a novel frameshift mutation was identified at nucleotide position 616, where adenine was duplicated (c.616dupA) (Fig. 1b). The corresponding amino acid sequence produced asparagine at position 206 instead of methionine with a termination codon at amino acid position 209 (p.M206Nfs"4), which resulted in a truncated protein. The patient was heterozygous for this mutation, and no other variations were found at other exons or intron-exon boundaries. To determine whether this mutation was pathogenic only for this patient, RFLP analysis was performed since the mutation destroyed the FokI site. This variation was not found in the 100 unrelated individuals with normal hearing (data not shown). Moreover, the multiple alignments of the amino acid sequence in several vertebrate species revealed that this amino acid position 206 was highly conserved among these species (Fig. 1c).

A non-synonymous variation at nucleotide position 232 was detected in the proband (III-1) of the SR-167 family (Fig. 2a). The proband had moderate hearing loss and the average threshold in the PTA was calculated as $61.7 \mathrm{~dB}$ for the right ear and $70 \mathrm{~dB}$ for the left ear. This single-nucleotide change produced guanine at nucleotide position 232 instead of adenine (c.232A > G) resulting in an isoleucine to valine (p.I78V) substitution (Fig. 2b). This variation was reported in the 1000 Genomes Project Database (SNP ID: rs141811441) with a global minor allele frequency (MAF) of $0.1 \%$. However, it was predicted to be tolerated (score of 0.59 ) by SIFT but probably damaging (score by 0.982 ) by PolyPhen in the 1000 Genomes Project Database and to be disease causing by the Mutation Taster program. To verify whether this variation is pathogenic, the proband's father (II-2) was screened. The results confirmed that this variation

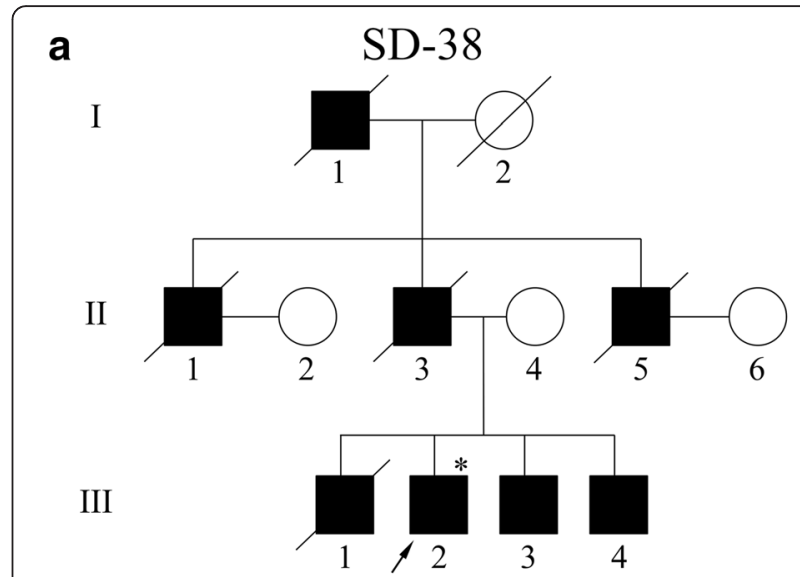

b

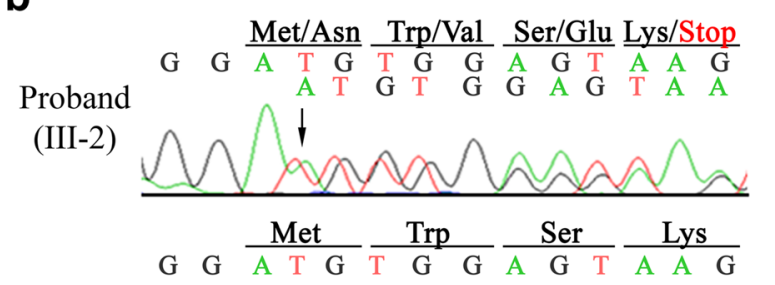

Normal

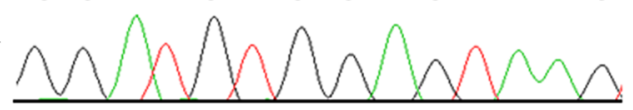

C Homo_sapiens CVRILQGLVE GVTYPACHGM WSKWAPPLER SRLATTSFCG 226 Aotus_nancymaae CVR ILQGLVE GVTYPACHGM WSKWAPPLER SRLATTSFCG 226
Bos_taurus CVIILQGLVE GVTYPACHGM WSKWAPPLER SRLATTSFCG 226 Mus_musculus GVRILQGLVE GVTYPACHGM WSKWAPPLER SRLATTSFCG 239 Rattus_norvegicus CVRILQGLVE GVTYPACHGM WSKWAPPLER SRLATTSFCG 226 Xenopus_tropicalls SVRVLQGLVE GVTYPACHGM WSKWAPPLER SRLATTSFCG 226 Danio_rerio FVRILQGLVE GVTYPACHGM WSKWAPPLER SRLATTSFCG 226 Consensus CVRILQGLVE GVTYPACHGM WSKWAPPLER SRLATTSFCG \begin{tabular}{c} 
Conservation \\
\hline Cos
\end{tabular}

Fig. 1 Mutation analysis of the SD-38 family with the p.M206 Nfs* 4 mutation. (a) Pedigree of Korean family SD-38. Squares and circles represent males and females, respectively. Filled symbols denote affected individuals, whereas unfilled symbols denote unaffected individuals. Diagonal lines through the symbols represent deceased members. The arrow indicates the proband of the SD-38 family. The individual evaluated in this study is marked with an asterisk. (b) Partial result of DNA sequencing analysis of the SLC17A8 gene for the proband (III-2) with p.M206Nfs*4 and a normal control. The wild type nucleotide sequence at position 616 is compared with that of the proband (III-2), which shows heterozygous c.616dupA, indicated with the arrow. (c) Alignment of the VGLUT3 amino acid sequence in different vertebrate species: Homo sapiens, Aotus nancymaae, Bos taurus, Mus musculus, Rattus norvegicus, Xenopus tropicalis, and Danio rerio. The arrow shows the position of the p.M206 Nfs*4 mutation

was not co-segregated (Fig. 2b). However, the multiple alignments of amino acid sequences showed that the amino acid position of this variation was a conserved region (Fig. 2c).

Another non-synonymous variation was identified at nucleotide position 1120 in the proband (III-2) of the SR357 family (c.1120G $>$ T) (Fig. 3a). This proband had 

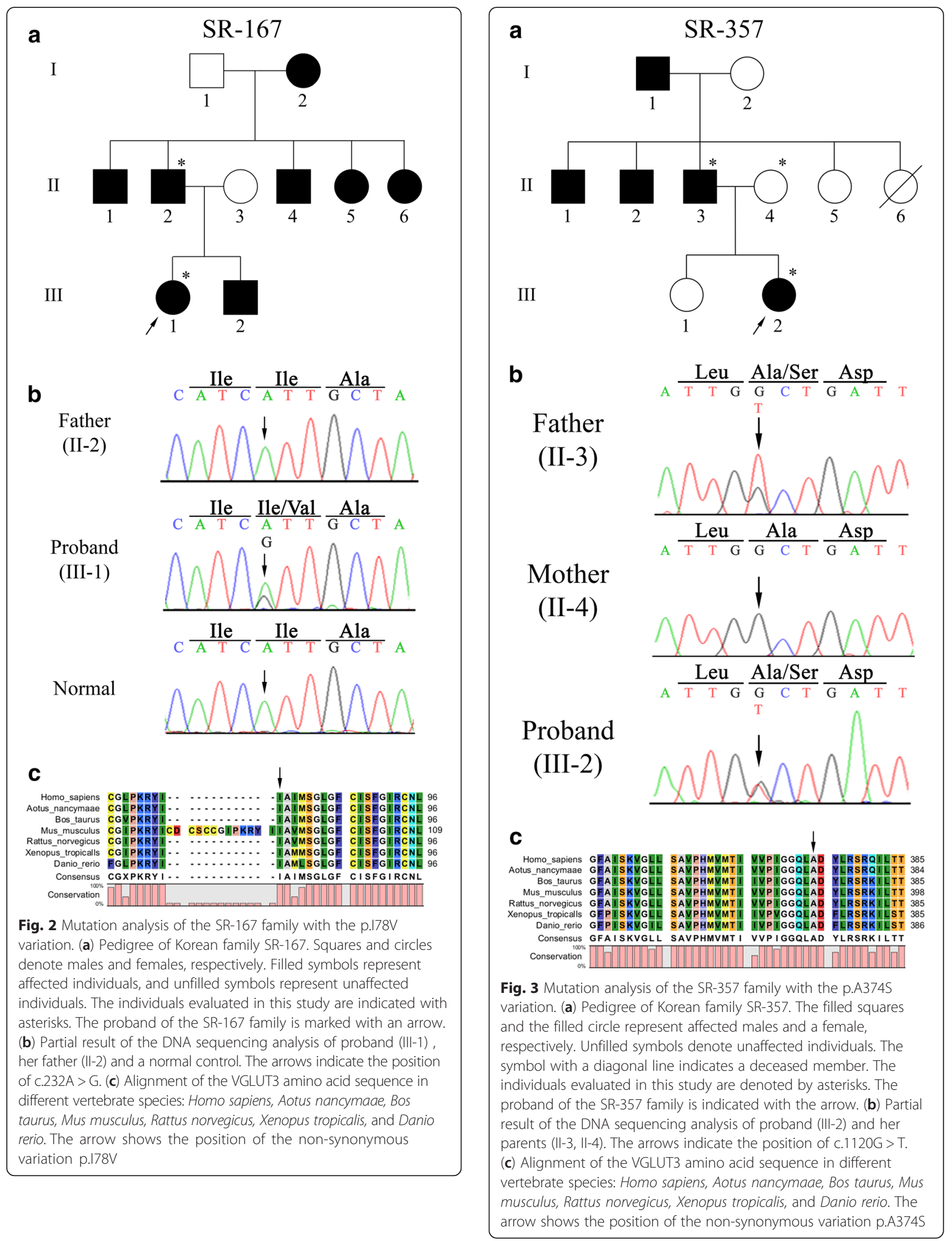
moderate hearing loss calculated the average threshold in the PTA as $54.2 \mathrm{~dB}$ for the right ear and $45.8 \mathrm{~dB}$ for the left ear. The variation changed guanine to thymine, which led to an alteration in the amino acid position at 374, wherein alanine was replaced with serine (p.A374S) (Fig. 3b). This variation has been described in the 1000 Genomes Project Database (SNP ID: rs138307707) with a MAF of $0.2 \%$. Moreover, it has been predicted to be deleterious (score of 0.01 ) by SIFT, probably damaging (score of 0.999 ) by PolyPhen, and disease causing by the Mutation Taster program. Therefore, the genes of the proband's parents (II-3, II-4) were screened, and the variation was revealed to be co-segregated from her father (II-3) (Fig. 3b). The position of this variation, p.A374S, was highly conserved based on the multiple alignments of amino acid sequences (Fig. 3c). However, 2 controls with normal hearing were heterozygous for this variation.

\section{Discussion}

Degeneration or loss of function of the cochlea, including the IHCs, is an important cause of SNHL $[5,6]$. VGLUT3, encoded by the $S L C 17 A 8$ gene, is expressed selectively in the IHCs [17]. The function of VGLUT3 is to transport glutamate into the synaptic vesicles, where glutamate plays an important role as an excitatory neurotransmitter $[13,15,17]$.

A novel pathogenic mutation, c.616dupA (p.M206Nfs"4), in the SLC17A8 gene was identified in a patient (III-2) of the SD-38 family. This mutation not only changed the neutral nonpolar amino acid methionine into the polar amino acid asparagine but also created a stop codon at amino acid position 209. According to molecular models, VGLUT3 is composed of 12 transmembrane helices, and position M206 is suggested to exist between transmembrane domains 4 and 5 as a topological domain and is probably located on the cytoplasmic side [15] (http://www.ncbi.nlm.nih.gov/protein/NP_647480.1). Therefore, this mutation could lead to the loss of the rest of the transmembrane domains (5 to 12), thereby resulting in a truncated protein. The truncated protein may cause malfunction or dysfunction of VGLUT3 in the IHCs, thereby leading to SNHL, but further functional studies are required to elucidate the effect of the mutation on this protein. Since neither the 1000 Genomes Project Database nor the dbSNP database had any information on this mutation and since this study is the first to describe it, we defined it as a novel mutation. Furthermore, the p.M206Nfs" 4 mutation was predicted as disease causing by the Mutation Taster program. These results support the theory that the novel p.M206Nfs"4 mutation is more likely to be a pathogenic mutation causing ADNSHL.

The non-synonymous variation p.I78V in the SR-167 family was not co-segregated from the father (II-2), and the patient (III-1) may have inherited this variation from the mother (II-3), who had normal hearing. Because isoleucine and valine are neutral nonpolar amino acids, the molecular structure of the protein may not be disturbed, and the effect of the amino acid substitution may be subtle. Therefore, this variation is considered a benign polymorphism rather than a disease-causing mutation.

The single-nucleotide change in the SR-357 family was identified at amino acid position 374 (p.A374S). Although this variation was co-segregated from the father (II-3) to the proband (III-2), we found that the 2 controls with normal hearing were heterozygous for this variation. Even though a neutral nonpolar amino acid, alanine, was changed to a polar amino acid, serine, this residue may be located at the edge of transmembrane domain 8 and away from the pore according to the molecular model of VGLUT3 [15], which would have less influence on the protein structure. Therefore, it is predicted that the chance of this variation being pathogenic is relatively low. However, further functional studies will be required to confirm this hypothesis.

In addition, a synonymous variation, c.171G $>\mathrm{A}$, was detected with a MAF of $1.03 \%$ in 18 patients out of 87 subjects in this study (data not shown). This variation has been reported with a MAF of $2.8 \%$ in the $1000 \mathrm{Ge}-$ nomes Project Database (SNP ID: rs11110359), including $1.4 \%$ in East Asians. Our result of $1.03 \%$ MAF was very similar to the East Asians.

\section{Conclusions}

In summary, this study is the first genetic analysis of SLC17A8 in patients with ADNSHL in Korea and reports a novel duplication mutation in the SLC17A8 gene. This study will help in predicting the primary cause of ADNSHL in Korean patients. In addition, further studies with more samples are required to improve our understanding of the SLC17A8 gene as the major causative gene for ADNSHL in Korea.

\section{Abbreviations \\ SNHL: Sensorineural hearing loss; SLC17A8: Solute carrier family 17, member 8; VGLUT3: Vesicular glutamate transporter 3; IHCs: Inner hair cells; ADNSHL: Autosomal dominant non-syndromic hearing loss; MFS: Major facilitator superfamily; PTA: Pure-tone audiometry; PCR: Polymerase chain reaction; RFLP: Restriction fragment length polymorphism; MAF: Minor allele frequency.}

\section{Competing interests}

The authors declare that they have no competing interests.

\section{Authors' contributions}

NR performed the experiments and contributed to the writing of the manuscript. NR, BS, and MAK analyzed the data. UKK, HJP, KYL, and JYC conceived and designed this study. UKK provided technical advice. UKK supervised the project and provided critical review of the manuscript. All authors read and approved the final manuscript.

\section{Acknowledgements}

Our research was supported by a grant of the Korea Health Technology R\&D Project through the Korea Health Industry Development Institute (KHIDI), 
funded by the Ministry of Health \& Welfare, Republic of Korea (HI14C2119 to U.K.K). This work was supported by the National Research Foundation of Korea (NRF) funded by the Ministry of Science, Information and Communications Technology (ICT) and Future Planning

(2015R1A2A2A01003438 to U.K.K).

\section{Author details}

'Department of Biology, College of Natural Sciences, Kyungpook National University, Daegu 41566, South Korea. ${ }^{2}$ Soree Ear Clinics, Seoul 06068, South Korea. ${ }^{3}$ School of Life Sciences, BK21 Plus KNU Creative BioResearch Group, Kyungpook National University, Daegu 41566, South Korea. ${ }^{4}$ Department of Otorhinolaryngology-Head and Neck Surgery, School of Medicine, Kyungpook National University, Daegu 41944, South Korea. ${ }^{5}$ Department of Otorhinolaryngology, Yonsei University College of Medicine, Seoul 03722, South Korea.

Received: 26 August 2015 Accepted: 8 January 2016

Published online: 22 January 2016

\section{References}

1. Morton NE. Genetic epidemiology of hearing impairment. Ann N Y Acad Sci. 1991;630:16-31.

2. Morton CC, Nance WE. Newborn hearing screening-a silent revolution. $N$ Engl J Med. 2006:354(20):2151-64.

3. Morton CC. Genetics, genomics and gene discovery in the auditory system. Hum Mol Genet. 2002:11(10):1229-40.

4. Smith RJ, Bale Jr JF, White KR. Sensorineural hearing loss in children. Lancet. 2005;365(9462):879-90.

5. Parker MA. Biotechnology in the treatment of sensorineural hearing loss: foundations and future of hair cell regeneration. J Speech Lang Hear Res. 2011;54(6):1709-31.

6. Nakagawa T. Strategies for developing novel therapeutics for sensorineural hearing loss. Front Pharmacol. 2014;5:206.

7. ACMG. Genetics Evaluation Guidelines for the Etiologic Diagnosis of Congenital Hearing Loss. Genetic Evaluation of Congenital Hearing Loss Expert Panel. ACMG statement. Genet Med. 2002;4(3):162-71.

8. Mahboubi H, Dwabe S, Fradkin M, Kimonis V, Djalilian HR. Genetics of hearing loss: where are we standing now? Eur Arch Otorhinolaryngol. 2012; 269(7):1733-45.

9. Thirlwall AS, Brown DJ, McMillan PM, Barker SE, Lesperance MM. Phenotypic characterization of hereditary hearing impairment linked to DFNA25. Arch Otolaryngol Head Neck Surg. 2003;129(8):830-5.

10. Park MK, Sagong B, Lee JD, Bae SH, Lee B, Choi KS, et al. A1555G homoplasmic mutation from A1555G heteroplasmic mother with Pendred syndrome. Int J Pediatr Otorhinolaryngol. 2014;78(11):1996-9.

11. Greene CC, McMillan PM, Barker SE, Kurnool P, Lomax MI, Burmeister M, et al. DFNA25, a novel locus for dominant nonsyndromic hereditary hearing impairment, maps to 12q21-24. Am J Hum Genet. 2001;68(1):254-60.

12. Van Camp G, Willems PJ, Smith RJ. Nonsyndromic hearing impairment: unparalleled heterogeneity. Am J Hum Genet. 1997;60(4):758-64.

13. Ruel J, Emery S, Nouvian R, Bersot T, Amilhon B, Van Rybroek JM, et al. Impairment of SLC17A8 encoding vesicular glutamate transporter-3, VGLUT3, underlies nonsyndromic deafness DFNA25 and inner hair cell dysfunction in null mice. Am J Hum Genet. 2008;83(2):278-92.

14. Fremeau Jr RT, Burman J, Qureshi T, Tran CH, Proctor J, Johnson J, et al. The identification of vesicular glutamate transporter 3 suggests novel modes of signaling by glutamate. Proc Natl Acad Sci U S A. 2002;99(22):14488-93.

15. Almqvist J, Huang Y, Laaksonen A, Wang DN, Hovmoller S. Docking and homology modeling explain inhibition of the human vesicular glutamate transporters. Protein Sci. 2007;16(9):1819-29.

16. Pao SS, Paulsen IT, Saier Jr MH. Major facilitator superfamily. Microbiol Mol Biol Rev. 1998:62(1):1-34

17. Seal RP, Akil O, Yi E, Weber CM, Grant L, Yoo J, et al. Sensorineural deafness and seizures in mice lacking vesicular glutamate transporter 3. Neuron. 2008;57(2):263-75.

18. Dror AA, Avraham KB. Hearing impairment: a panoply of genes and functions. Neuron. 2010;68(2):293-308.

19. Stelma F, Bhutta MF. Non-syndromic hereditary sensorineural hearing loss: review of the genes involved. J Laryngol Otol. 2014;128(1):13-21.
20. Takamori S, Malherbe P, Broger C, Jahn R. Molecular cloning and functional characterization of human vesicular glutamate transporter 3. EMBO Rep. 2002;3(8):798-803.

21. Kim MA, Kim YR, Sagong B, Cho HJ, Bae JW, Kim J, et al. Genetic analysis of genes related to tight junction function in the Korean population with nonsyndromic hearing loss. PLoS One. 2014;9(4):e95646.

22. Kim YR, Kim MA, Sagong B, Bae SH, Lee HJ, Kim HJ, et al. Evaluation of the contribution of the EYA4 and GRHL2 genes in Korean patients with autosomal dominant non-syndromic hearing loss. PLoS One. 2015;10(3): e0119443.

23. Lee KY, Choi SY, Bae JW, Kim S, Chung KW, Drayna D, et al. Molecular analysis of the GJB2, GJB6 and SLC26A4 genes in Korean deafness patients. Int J Pediatr Otorhinolaryngol. 2008;72(9):1301-9.

\section{Submit your next manuscript to BioMed Central and we will help you at every step:}

- We accept pre-submission inquiries

- Our selector tool helps you to find the most relevant journal

- We provide round the clock customer support

- Convenient online submission

- Thorough peer review

- Inclusion in PubMed and all major indexing services

- Maximum visibility for your research

Submit your manuscript at www.biomedcentral.com/submit
) Biomed Central 